\title{
Developmental Dyspraxia
}

National Institute of Neurological Disorders and Stroke (NINDS)

\section{Source}

National Institute of Neurological Disorders and Stroke (NINDS). Developmental

Dyspraxia Information Page.

Developmental dyspraxia is a disorder characterized by an impairment in the ability to plan and carry out sensory and motor tasks. Generally, individuals with the disorder appear "out of sync" with their environment. Symptoms vary and may include poor balance and coordination, clumsiness, vision problems, perception difficulties, emotional and behavioral problems, difficulty with reading, writing, and speaking, poor social skills, poor posture, and poor short-term memory. Although individuals with the disorder may be of average or above average intelligence, they may behave immaturely. 